ISSN 1112-9867

Available online at http://www.jfas.info

\title{
DROUGHTS IN SEMI-ARID CEREAL REGIONS OF ALGERIA
}

\author{
D. Smadhi ${ }^{1, *}$, L. Zella $^{2}$ and H. Bachir ${ }^{1}$ \\ ${ }^{1}$ INRA, Division de Bioclimatologie et Hydraulique Agricole, Alger, Algérie \\ ${ }^{2}$ Université de Saâid Dahleb de Blida, Faculté Agro-Vétérinaire, Algérie
}

Received: 02 November 2016 / Accepted: 28 April 2017 / Published online: 01 May 2017

\begin{abstract}
The droughts in the semi-arid Algeria's cereal regions are evaluated by studying the average monthly rainfall, from September to June, over the period (1941-2011), based on the firstorder Markov chain. This method traces rainy and dry days, cumulative rainfall and the probability of receiving rainfall above the thresholds of $0,1,5,10,20$ and $30 \mathrm{~mm}$, in conjunction with the cereal development cycle. The rains analysed showed that the distribution of rainy days at the chosen thresholds did not exceed the variable averages of 6 to 9 days per month. These days are characterized by random cumulative rainfall, with probabilities fluctuating between $+40 \%$ and $+80 \%$. Hard droughts affect seeding dates and yield factors for rainfed cereals.
\end{abstract}

Keywords: Algeria, rainfall, Sowing, Markov Chain

Author Correspondence, e-mail: dalsmadhi@yahoo.fr

doi: http://dx.doi.org/10.4314/jfas.v9i2.29

\section{INTRODUCTION}

La céréaliculture pluviale en régions semi-arides d'Algérie, évolue sous une pluviométrie annuelle moyenne variant de 300 à $630 \mathrm{~mm} / \mathrm{an}$. Bien que ces lames d'eaux reflètent des quantités théoriquement suffisantes pour combler les besoins des céréales, leurs corrélations à la production céréalière annuelle, n'expliquent pas toute la prétendue influence [1]. En effet, ce n'est pas tant la pluviométrie annuelle moyenne qui importe mais surtout, sa répartition spatio-temporelle. En Tunisie, Yadh [2] a montré qu'en dépit d'une pluviométrie annuelle théoriquement, satisfaisante, le nombre de jours pluvieux, caractérisé par un déficit de 100 mm durant le cycle végétatif, a soldé un recul de la production de $20 \%$. Un jour pluvieux est 
en effet, définit par la disponibilité de $60 \%$ à $80 \%$ de la pluie brute [3]. Hills et Morgan [4] précisent qu'une journée est pluvieuse, si la lame d'eau précipitée est égale ou dépasse les 5 $\mathrm{mm}$. L'efficacité de la pluie est liée à la notion de seuils, mais également, à sa durée et sa probabilité d'occurrence. Ces notions peuvent définir des sècheresses agricoles selon Davey et al. [5] ; Sivakumar [6]. Des travaux dans ce sens, ont été réalisé en Afrique Central [7, 8], en Afrique de l'Ouest [6, 9], en France [10] et en Tunisie [11 et 12].

En Algérie, beaucoup d'études sont consacrées à la pluviométrie [13, 14], mais sont très rares $[15,16$ et 17] celles qui s’intéressent à son évolution en liaison avec la croissance des céréales emblavées, dominée par le blé dur, le blé tendre, l'orge et l'avoine.

Dans cette perspective, l'étude en cours, a pour objectif d'analyser la sécheresse agricole sur la base de la pluviométrie mensuelle, tant à l'échelle spatiale que temporelle. Le modèle stochastique basé sur la chaine de Markov [18, 19, 20], est utilisé dont le but d'évaluer l'évolution des variables afférentes à ce facteur. Il s'agit du nombre de jours de pluies, la durée et les probabilités de sécheresses qui condamnent partiellement, la production et la productivité céréalière. Ces variables sont peu étudiées en raison probablement, de la difficulté d'acquérir des données de mesures quotidiennes, fiables et comportant peu de lacunes.

\section{MATERIELS ET METHODES}

\subsection{Aire d'étude}

L'étude couvre les régions semi-arides comprises entre $32^{\circ}$ et $37^{\circ}$ de latitude Nord et entre $-1^{\circ}$ et $9^{\circ}$ de longitude Est (figure 1$)$. La région Est (RE), la région Centre (RC) et la région Ouest (RO). Ces régions s'étendent sur une surface de 21,5 Mha, soit 10\% du territoire Nord de l'Algérie (NA) et sont limitées administrativement, en wilayas (ou unités territoriales) céréalières de proportions variables. Un gradient pluviométrique annuel latitudinal, décroissant d'Est en Ouest (420, 405 et $345 \mathrm{~mm}$ ), caractérise, ces régions [1]. 


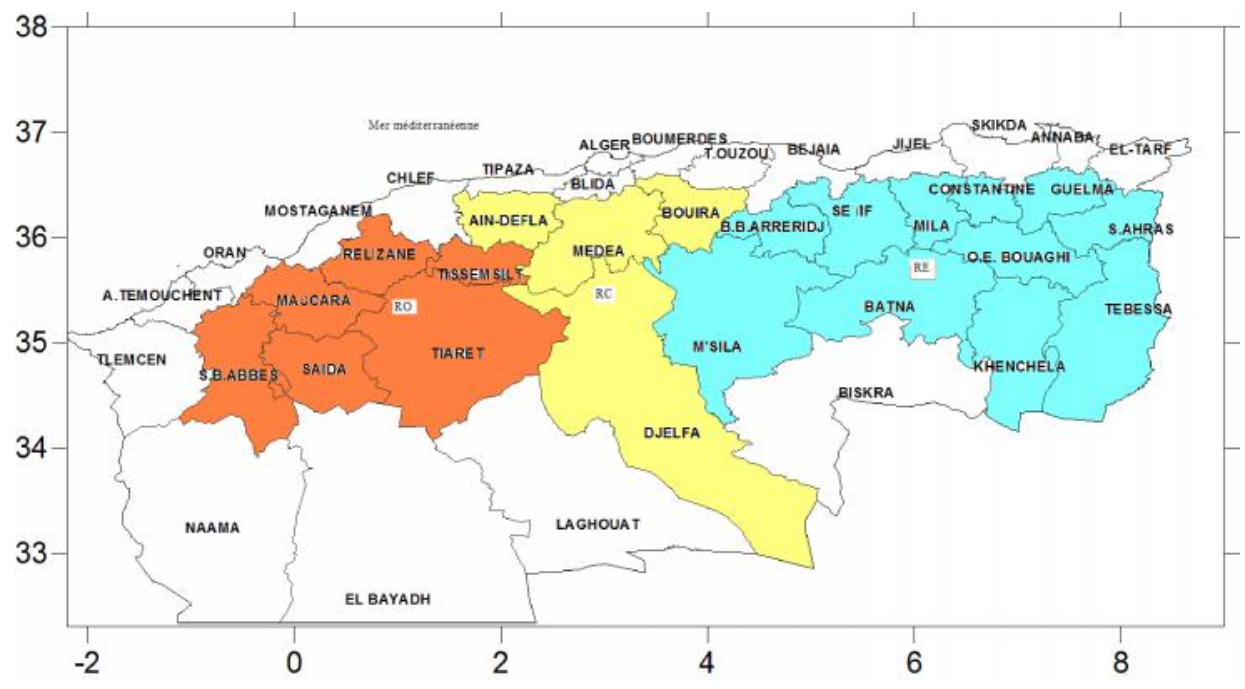

Fig 1. Localisation géographique des régions semi-arides d'Algérie. Echelle 1/40 000000.

\subsection{Réseau de stations et données pluviométriques}

Les stations pluviométriques en nombre de 76 , sont parsemées au niveau des wilayas céréalières semi-arides. Ces stations appartiennent au réseau de l'Office nationale de la météorologie (ONM) et à celui de l'Agence nationale de la ressource Hydraulique (ANRH).

Les données de pluies brutes recueillies, ont été extraites à l'échelle journalière (24 heures), sur une période de 70 ans (1940-2011). Cette échelle permet de diagnostiquer les jours pluvieux (jp), les jours secs (js), le début et la fin des pluies en rapport avec les quantités reçues. Le pas de temps choisi, est mensuel $\left(\mathrm{P}_{\mathrm{M}}\right)$, de septembre $(\mathrm{S})$ à juin $(\mathrm{Ju})$. Ce pas considère la pluviométrie aux seuils supérieurs, $\mathrm{x} \mathrm{mm}$, noté $\mathrm{P}_{\mathrm{x} \mathrm{mm}}$, intégrant la distribution spatio-temporelle.

Les seuils considérés, sont représentés par des $\mathrm{P}_{\searrow 1,1 \mathrm{~mm}}, \mathrm{P}_{25 \mathrm{~mm}}, \mathrm{P}_{\searrow 10 \mathrm{~mm}}, \mathrm{P}_{20 \mathrm{~mm}}$ et $\mathrm{P}_{230 \mathrm{~mm}}$. Le premier seuil est retenu pour caractériser les pluies météorologiques moyennes de $\mathrm{S}$ à Ju. Le deuxième seuil est utilisé pour caractériser les pluies agricoles. Les trois derniers seuils, renseignent sur la satisfaction des besoins en eau des céréales, depuis le semis jusqu'à la maturation des grains. Ces seuils participent à différencier selon Heathcote [21] et El Hassani [22], les pluies météorologiques et agronomiques.

\subsection{Evaluation statistique}

\subsubsection{Jours pluvieux et cumuls pluviométriques}

Les pluies régionales, sont analysées en référence au modèle stochastique à cinq variables, dont l'approche repose sur la démarche de la chaîne de Markov d'ordre 1. La démarche 
permet de calculer l'évolution mensuelle des jours pluvieux (jp) et secs (js) ; et quantifier les cumuls de pluies reçues, selon que les jours pluvieux sont alternés avec les jours secs.

Les séries de données pluviométriques journalières utilisées, sont simulées à une matrice composée de deux états : un état pluvieux et un état sec. Le premier représente toutes les valeurs de pluies égales ou supérieures aux seuils pluviométriques, $\mathrm{P}_{× \mathrm{xmm}}$, il est représenté par le chiffre 1. Le deuxième désigne toutes les valeurs inférieures à ces seuils, il est signalé par le chiffre 0 . Les matrices des séries de pluies nouvellement conçues, ne comportent alors que les valeurs égales à un (1) et à zéro (0), en rappelant que pour une chaîne de Markov d'ordre 1, l'état de la variable (pluie) à l'instant $(t)$ ne dépend que de son état à l'instant ( $t$-1) [20]. Les probabilités d'occurrences des cumuls pluviométriques et les sécheresses qui caractérisent les mois céréaliers sont ainsi, évaluées. Ces paramètres participent à simuler les dates de semis ainsi, que les autres facteurs d'élaboration de rendements des céréales (de la germination à la maturation des grains) en régime pluvial, dans les régions d'étude.

Les calculs itératifs allant de simples moyennes jusqu'à la résolution de système d'équations complexes, sont facilités par l'utilisation du logiciel Instat version 3.7, bien adapté selon Sivakumar et al. [9] au calcul d'événements climatiques.

\subsubsection{Probabilités des cumuls pluviométriques et des sécheresses}

Les probabilités des cumuls pluviométriques moyens, sur la période d'étude, à l'échelle régionale, sont évaluées en posant cinq (5) hypothèses. La première porte sur la meilleure occurrence pour chaque mois, de recevoir les premières $\mathrm{P}_{\searrow 1,1 \mathrm{~mm}}$. La seconde, la troisième, la quatrième et la cinquième hypothèse, représentent respectivement les meilleures occurrences de recevoir durant tous les mois, les premières $\mathrm{P}_{25 \mathrm{~mm}}, \mathrm{P}_{\searrow 10 \mathrm{~mm}}, \mathrm{P}_{20 \mathrm{~mm}}$ et $\mathrm{P}_{230 \mathrm{~mm}}$, sur l'année moyenne. L'occurrence des cumuls de pluies, est évaluée aux probabilités de répartition de $20 \%, 30 \%, 50 \%$ et $80 \%$ en relation avec la définition des sécheresses.

Les sécheresses représentent les mois où, il a été recueilli des quantités de pluies inférieures aux seuils définis et sont calculées également, aux probabilités de 20\%, 30\%, 50\% et $80 \%$. Ces pourcentages permettent d'estimer, la ténacité de la culture céréalière à des séquences sèches équivalente à 10,15 et 20 jours au cours de chaque mois.

\subsubsection{Date de semis}

Pour simuler les dates de semis des céréales en pluviales, le modèle a été appliqué à titre d'exemple aux wilayas de Batna, Bordj Bou Arreirdj, Constantine, Guelma, Souk Ahras, Sétif dans la RE et à Tiaret dans la RO. La démarche basée sur les paramètres évoqués ci-dessus, défalque de plus, une quantité de $40 \%$ de la lame pluviométrique mensuelle. Cette défalcation permet d'estimer la réserve d'eau dans les 15 premiers cm du sol durant les 10, 15 et 20 jours. 
Les calculs sont réalisés, en référence à des dates de semis fixées au départ, simulant un semis précoce, intermédiaire ou tardif.

Pour chaque scénario choisi (10, 15 et 20 jours), le modèle cherche la première date possible pour réussir un semis avec une humidité du sol supérieur à $5 \mathrm{~mm}$. Lorsque, cette condition n'est pas remplie, une autre date de semis est recherchée pour laquelle la somme des pluies des séquences considérée, est supérieure à $10 \mathrm{~mm}$. Dans le cas, où le test est négatif, l'itération de test de semis est répétée pour des cumuls de 20 puis de $30 \mathrm{~mm}$.

Les dates obtenues, sont différenciées par l'analyse du test de Student en posant deux hypothèses. La première considère, des dates identiques en relation avec les événements climatiques similaires; la deuxième considère, des dates différentes en liaison avec les événements climatiques distincts ; avec un degré de liberté (DDL) $=\mathrm{N}_{1}+\mathrm{N}_{2}-2\left(\mathrm{~N}_{1}\right.$ : la taille du $1^{\text {er }}$ groupe des dates $; \mathrm{N}_{2}$ : la taille du $2^{\text {eme }}$ groupe des dates; $\mathrm{T}_{\text {critique }}=1,73$ au niveau de signification 0,05$)$.

\section{RÉSULTATS ET DISCUSIONS}

\subsection{Nombre de jours pluvieux}

L'évolution des jours pluvieux au seuil de $P_{\searrow, 1 \mathrm{~mm}}$, sur une moyenne de 70 ans, dans les régions semi-arides, se produit à des temps variables et n'apparait pas sur les courbes de variations mensuelles, tracées à partir des hauteurs moyennes de chaque jour, du mois (figure 2). En $\mathrm{S}, \mathrm{O}, \mathrm{N}$ et $\mathrm{M}, \mathrm{A}, \mathrm{Ma}$, le nombre de jp moyen, est relativement équivalent à 6 et 8 $\mathrm{j} /$ mois. Il atteint $9 \mathrm{j}$ durant les mois d'hiver (D, J, F).

Au seuil de $5 \mathrm{~mm}$, le nombre de jp par mois dépasse à 4 ou 5 jp et aux seuils de 10, 20 et 30 $\mathrm{mm}$, il devient négligeable (<3). Les js sont alors, dominants le long des mois dans l'ensemble des régions. Ce constat ne conclue cependant, pas à l'existence de périodes sèches continuent durant les mois agricoles. 


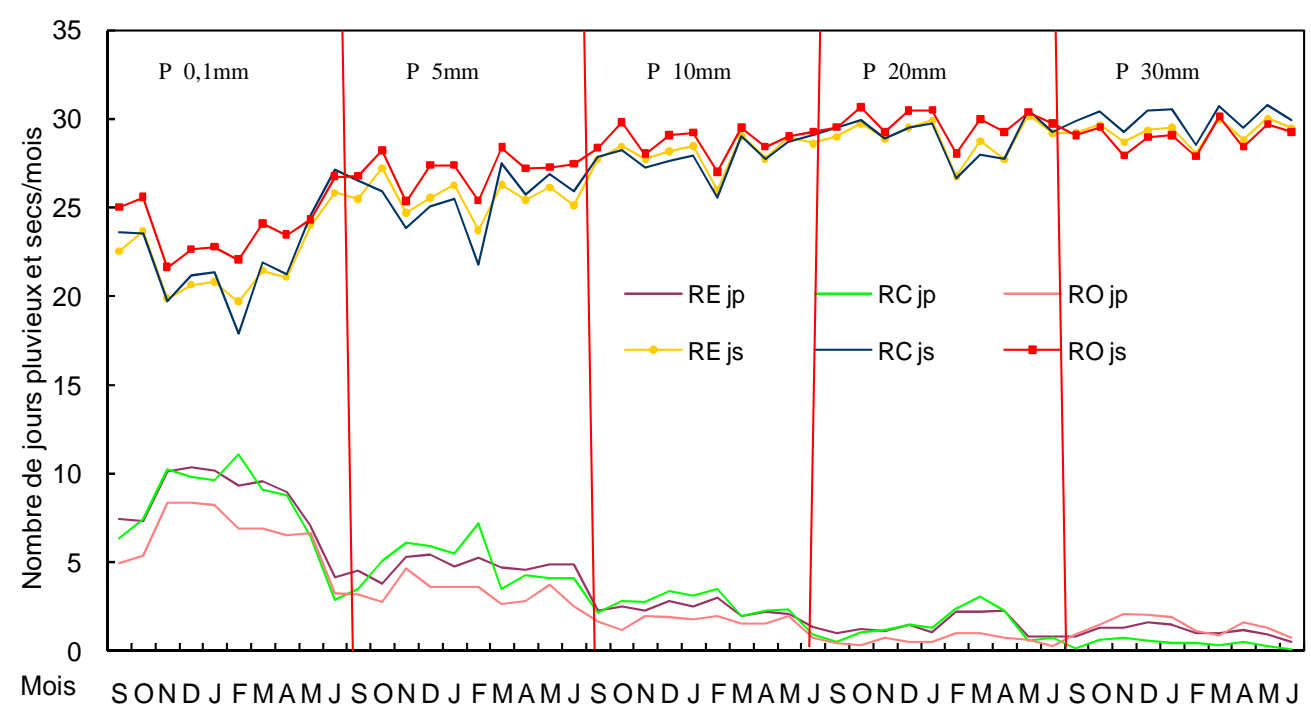

Fig. 2. Evolution mensuelle du nombre de jours pluvieux (jp) et secs (js) aux seuils de pluies supérieurs à $0,1,5,10,20$ et $30 \mathrm{~mm}$ dans les régions semi-arides.

\subsection{Cumuls pluviométriques}

L'évolution des cumuls pluviométriques moyens dans la RE, la RC et la RO, se distingue par les dates d'installation des $\mathrm{P}_{\searrow 1,1 \mathrm{~mm}}, \mathrm{P}_{25 \mathrm{~mm}}, \mathrm{P}_{\searrow 10 \mathrm{~mm}}, \mathrm{P}_{\geq 20 \mathrm{~mm}}$ et $\mathrm{P}_{\geq 30 \mathrm{~mm}}$ de $\mathrm{S}$ à $\mathrm{Ju}$.

Au premier seuil, la figure 3 montre des pluies dès $\mathrm{S}$ avec des cumuls moyens de $31 \mathrm{~mm}$ (RE), $22 \mathrm{~mm}(\mathrm{RC})$ et $17 \mathrm{~mm}(\mathrm{RO})$. Ces valeurs qui atteignent respectivement, des pics d'environ $59 \mathrm{~mm}, 63 \mathrm{~mm}$ et $49 \mathrm{~mm}$ en décembre (D), diminuent au fur et à mesure, relevant des valeurs minimums (12 à $19 \mathrm{~mm}$ ) en Ju marquant, la fin de la saison pluviale. A ce seuil, les premières averses ne montrent pas le début des pluies en liaison avec l'installation de la céréaliculture dans les régions d'étude.

Au second seuil, et selon la même figure 3, l'évolution des pluies mensuelles, suit celle des pluies précédentes. $\mathrm{S}$ indique une fois encore, le début des pluies mais avec des cumuls moyens qui ne dépassent pas, $18 \mathrm{~mm}$. D, janvier $(\mathrm{J})$ et février $(\mathrm{F})$, ces mois qui correspondent aux tallages des céréales, caractérisent des cumuls pluviométriques, voisin de $42 \mathrm{~mm}$. Quant aux mois de mars (M), avril (A) et mai (Ma), ils ne cumulent que le $1 / 4$ des pluies significatives, soit $25 \mathrm{~mm}$ qui contribuent à l'élaboration des rendements céréaliers. A cette échelle de temps et d'espace, Ju avec une moyenne de $9 \mathrm{~mm}$ seulement, marque la fin des pluies de la saison agricole.

Aux trois derniers seuils, les cumuls mensuels diminuent graduellement. Au seuil de $\mathrm{P}_{\geq 10 \mathrm{~mm}}$, les cumuls de pluies de $\mathrm{S}, \mathrm{O}, \mathrm{N}$ et $\mathrm{M}, \mathrm{A}$, Ma sont proches ou égales au seuil considéré et sont doublés, en D, J et F. Aux seuils de $\_20 \mathrm{~mm}$ et $\mathrm{P}_{230 \mathrm{~mm}}$, les cumuls mensuels sont relativement 
négligeables $(<2 \%)$ dans l'ensemble des régions.

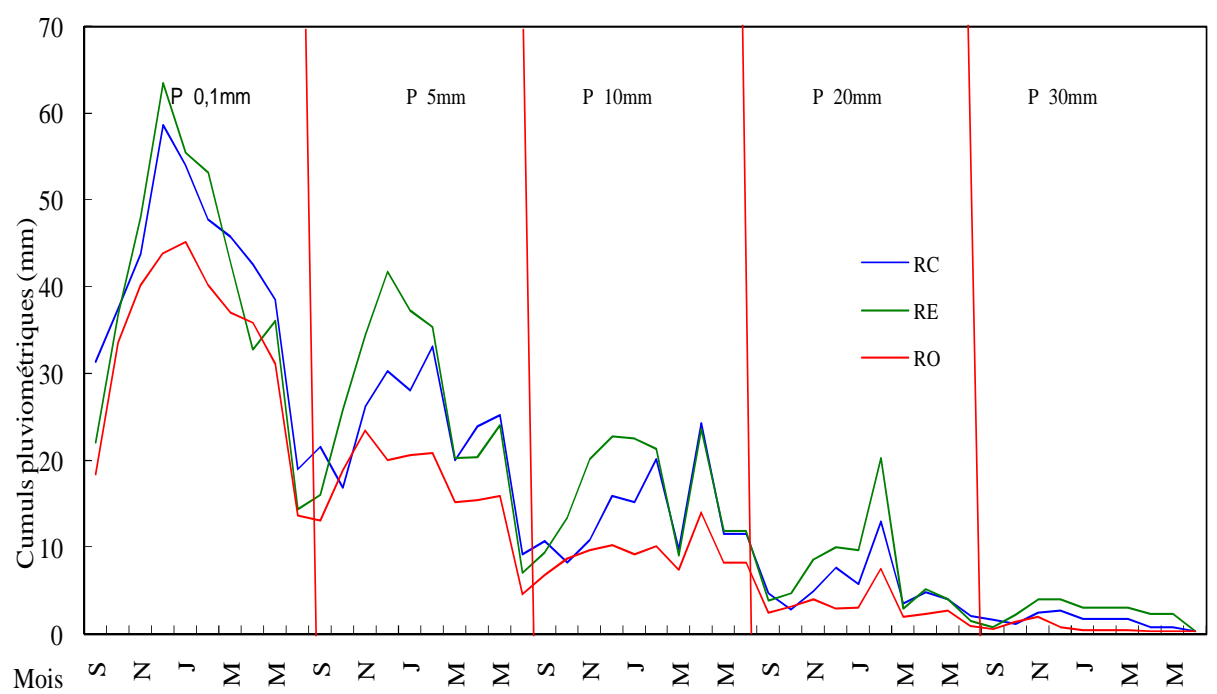

Fig. 3. Evolution mensuelle des cumuls pluviométriques moyens, aux seuils supérieurs de 0,1, $5,10,20$ et $30 \mathrm{~mm}$ dans les régions semi-arides céréalières.

\subsection{Probabilités des pluies significatives}

Les probabilités de recevoir des pluies significatives de $\mathrm{S}$ à Ju selon l'allure de la figure 4, sont variables d'une région à l'autre selon les pourcentages de répartition de 20\%, 30\%, 50\% et $80 \%$. Ces probabilités de l'ordre de $+20 \%$ à $+80 \%$ permettent la préparation des sols $(\mathrm{S})$, des semis $(\mathrm{O})$, des germinations $(\mathrm{N})$ et des paramètres d'élaboration des rendements des céréales (M, A, Ma). Les pluies significatives restent néanmoins plus probables, aux pourcentages les plus élevés $(+80 \%)$.

Ces résultats corroborent ceux obtenus précédemment, confirmant une carence en eau durant le cycle de développement des céréales, dans les régions semi-arides. 


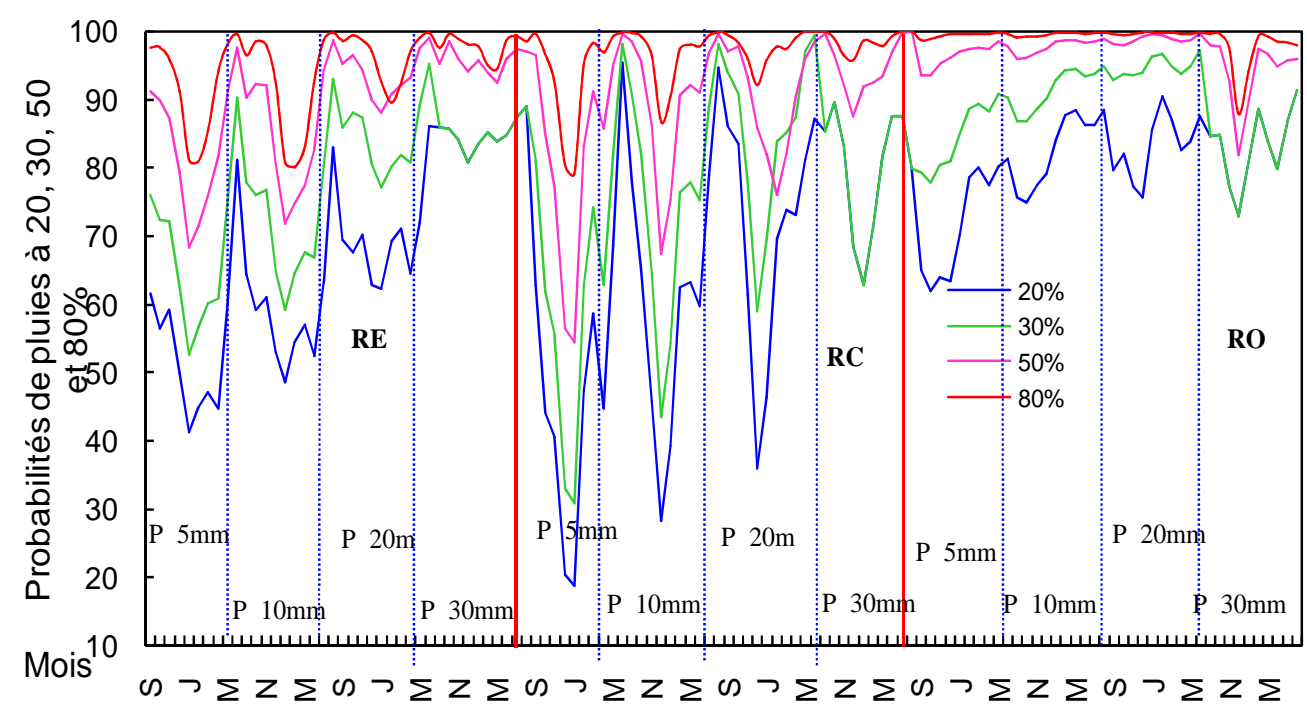

Fig. 4. Probabilités d'occurrences des pluies mensuelles supérieures à 5, 10, 20 et $30 \mathrm{~mm}$

\subsection{Probabilités de sècheresses}

La figure 5 confirme les risques d'une céréaliculture pluviale, dans les régions d'étude ; en effet, les probabilités de séquences sèches de plus de 10 et 15 jours au cours des 30 jours de chaque mois, peuvent atteindre jusqu'à $+80 \%$, quelque soit le seuil pluviométrique. Elles diminuent néanmoins, à partir du vingtième jour (20 j).

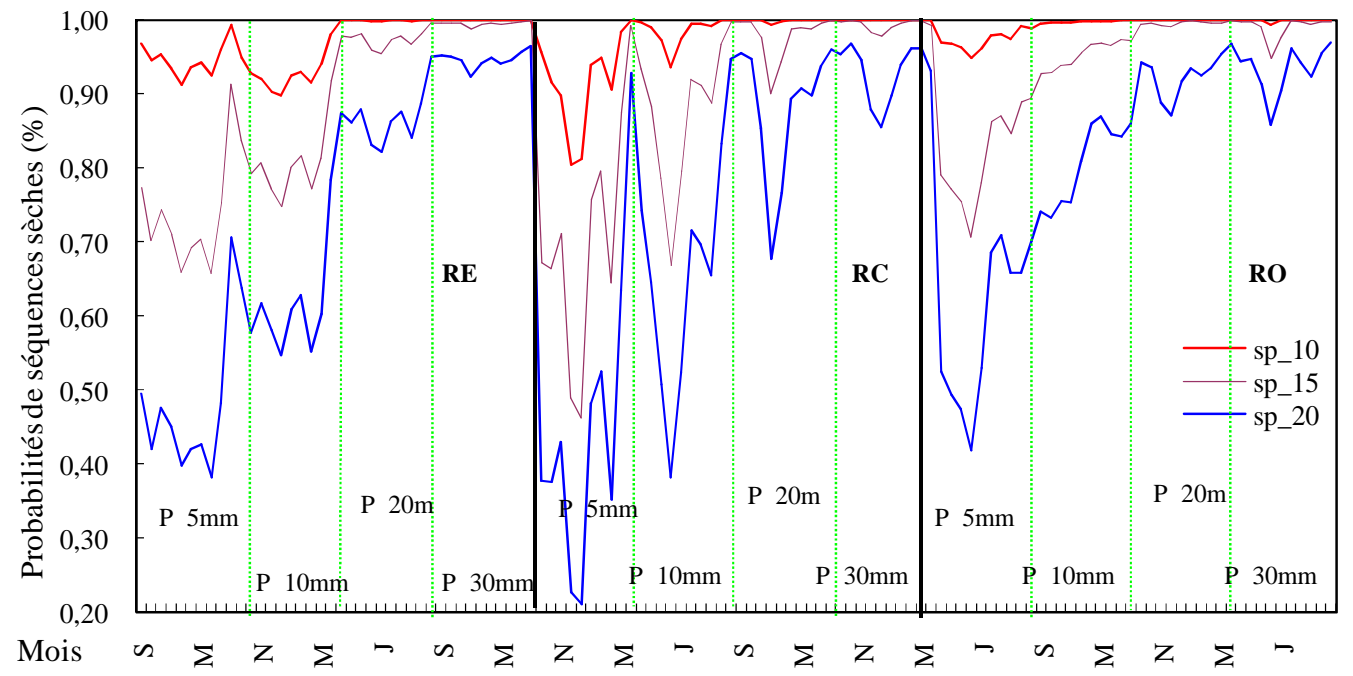

Fig. 5. Probabilités de séquences sèches aux seuils supérieurs de 5, 10, 20 et $30 \mathrm{~mm}$ dans les régions d'études

\subsection{Date de semis}

Un ensemble de dates qui reflètent un semis précoce, intermédiaire et tardif propres aux wilayas céréalières, semi-arides, a été généré pour la période d'étude. Les résultats synthétisés 
dans le tableau 1, ont montré que, quelques soient les dates simulées par le modèle en référence aux dates d'entrées au seuil significatif de 0,05, aucun événement pluviométrique (occurrence de pluie) particulier, n'agirait indistinctement sur la période de la floraison céréalière.

Tableau 1. Dates de semis des céréales pluviales proposées par le modèle Pluviométrique en référence aux dates fixées en entrée

\begin{tabular}{|c|c|c|c|c|c|c|c|c|c|c|}
\hline Wilayas & D.S.R & 50 & $15 \mathrm{O}$ & $25 \mathrm{O}$ & $4 \mathrm{~N}$ & $14 \mathrm{~N}$ & $24 \mathrm{~N}$ & $4 \mathrm{D}$ & $14 \mathrm{D}$ & $24 \mathrm{D}$ \\
\hline & $\mathrm{Mx}$ & Sèche & Sèche & Sèche & Sèche & Sèche & sèche & Sèche & sèche & Sèche \\
\hline \multirow[t]{2}{*}{ Batna } & M & $25 \mathrm{D}$ & $14 \mathrm{~J}$ & $14 \mathrm{~J}$ & $28 \mathrm{~J}$ & $18 \mathrm{~F}$ & $18 \mathrm{~F}$ & $18 \mathrm{~F}$ & $18 \mathrm{~F}$ & $18 \mathrm{~F}$ \\
\hline & SD & $5 \mathrm{~J}$ & $5 \mathrm{~J}$ & $5 \mathrm{~J}$ & $14 \mathrm{~J}$ & $26 \mathrm{~J}$ & $26 \mathrm{~J}$ & $26 \mathrm{~J}$ & $26 \mathrm{~J}$ & $26 \mathrm{~J}$ \\
\hline Bordj Bou & $\mathrm{Mx}$ & Sèche & Sèche & Sèche & Sèche & Sèche & sèche & Sèche & sèche & Sèche \\
\hline \multirow[t]{3}{*}{ Arreirdj } & M & $28 \mathrm{~J}$ & $13 \mathrm{~F}$ & $05 \mathrm{M}$ & $05 \mathrm{M}$ & $18 \mathrm{M}$ & $19 \mathrm{~A}$ & $19 \mathrm{~A}$ & $19 \mathrm{~A}$ & $19 \mathrm{~A}$ \\
\hline & SD & $15 \mathrm{~J}$ & $15 \mathrm{~J}$ & $20 \mathrm{~J}$ & $22 \mathrm{~J}$ & $26 \mathrm{~J}$ & $07 \mathrm{~J}$ & $07 \mathrm{~J}$ & $07 \mathrm{~J}$ & $07 \mathrm{~J}$ \\
\hline & Mx & Sèche & Sèche & Sèche & Sèche & Sèche & sèche & Sèche & sèche & Sèche \\
\hline \multirow[t]{3}{*}{ Constantine } & M & $30 \mathrm{D}$ & $02 \mathrm{~J}$ & $06 \mathrm{~J}$ & $11 \mathrm{~J}$ & $17 \mathrm{~J}$ & $25 \mathrm{~J}$ & $28 \mathrm{~J}$ & $18 \mathrm{~J}$ & $04 \mathrm{M}$ \\
\hline & SD & $15 \mathrm{~N}$ & $14 \mathrm{~N}$ & $12 \mathrm{~N}$ & $09 \mathrm{~N}$ & $09 \mathrm{~N}$ & $08 \mathrm{~N}$ & $07 \mathrm{~N}$ & $15 \mathrm{~N}$ & $16 \mathrm{~N}$ \\
\hline & $\mathrm{Mx}$ & $14 \mathrm{~F}$ & $14 \mathrm{~F}$ & $14 \mathrm{~F}$ & $14 \mathrm{~F}$ & $14 \mathrm{~F}$ & $14 \mathrm{~F}$ & $14 \mathrm{~F}$ & $14 \mathrm{~F}$ & $14 \mathrm{~F}$ \\
\hline \multirow[t]{3}{*}{ Guelma } & $\mathrm{M}$ & $02 \mathrm{D}$ & $02 \mathrm{D}$ & $06 \mathrm{D}$ & $06 \mathrm{D}$ & $07 \mathrm{D}$ & 013 D & $017 \mathrm{D}$ & $29 \mathrm{D}$ & $02 \mathrm{~J}$ \\
\hline & SD & $14 \mathrm{O}$ & $14 \mathrm{O}$ & $13 \mathrm{O}$ & $13 \mathrm{O}$ & $13 \mathrm{O}$ & $13 \mathrm{O}$ & $13 \mathrm{O}$ & $17 \mathrm{O}$ & $22 \mathrm{O}$ \\
\hline & Mx & $29 \mathrm{~J}$ & $29 \mathrm{~J}$ & $09 \mathrm{~A}$ & $09 \mathrm{~A}$ & $09 \mathrm{~A}$ & $09 \mathrm{~A}$ & $09 \mathrm{~A}$ & $09 \mathrm{~A}$ & $09 \mathrm{~A}$ \\
\hline \multirow[t]{3}{*}{ Souk Ahras } & M & $03 \mathrm{D}$ & $04 \mathrm{D}$ & $21 \mathrm{D}$ & $21 \mathrm{D}$ & $24 \mathrm{D}$ & $01 \mathrm{~J}$ & $05 \mathrm{~J}$ & $16 \mathrm{~J}$ & $09 \mathrm{~J}$ \\
\hline & SD & $06 \mathrm{O}$ & $06 \mathrm{O}$ & $20 \mathrm{O}$ & $20 \mathrm{O}$ & $17 \mathrm{O}$ & $13 \mathrm{O}$ & $09 \mathrm{O}$ & $06 \mathrm{O}$ & $28 \mathrm{O}$ \\
\hline & Mx & $12 \mathrm{~J}$ & $12 \mathrm{~J}$ & $12 \mathrm{~J}$ & $12 \mathrm{~J}$ & $12 \mathrm{~J}$ & $12 \mathrm{~J}$ & $12 \mathrm{~J}$ & $12 \mathrm{~J}$ & $12 \mathrm{~J}$ \\
\hline \multirow[t]{3}{*}{ Sétif } & $\mathrm{M}$ & $03 \mathrm{~N}$ & $03 \mathrm{~N}$ & $03 \mathrm{~N}$ & $05 \mathrm{~N}$ & $06 \mathrm{~N}$ & $06 \mathrm{~N}$ & $06 \mathrm{~N}$ & $06 \mathrm{~N}$ & $06 \mathrm{~N}$ \\
\hline & SD & $06 \mathrm{~N}$ & $06 \mathrm{~N}$ & $06 \mathrm{~N}$ & $06 \mathrm{~N}$ & $04 \mathrm{D}$ & $04 \mathrm{D}$ & $04 \mathrm{D}$ & $04 \mathrm{D}$ & $04 \mathrm{D}$ \\
\hline & $\mathrm{Mx}$ & $31 \mathrm{~J}$ & $31 \mathrm{~J}$ & $31 \mathrm{~J}$ & $31 \mathrm{~J}$ & $31 \mathrm{~J}$ & $31 \mathrm{~J}$ & $20 \mathrm{M}$ & $20 \mathrm{M}$ & $20 \mathrm{M}$ \\
\hline \multirow[t]{2}{*}{ Tiaret } & M & $13 \mathrm{~N}$ & $25 \mathrm{~N}$ & $26 \mathrm{~N}$ & $27 \mathrm{~N}$ & $2 \mathrm{D}$ & $20 \mathrm{~N}$ & $25 \mathrm{D}$ & $25 \mathrm{D}$ & $09 \mathrm{~J}$ \\
\hline & SD & $23 \mathrm{~S}$ & $25 \mathrm{~S}$ & $27 \mathrm{~S}$ & $28 \mathrm{~S}$ & $03 \mathrm{O}$ & $11 \mathrm{O}$ & $03 \mathrm{O}$ & $14 \mathrm{O}$ & $16 \mathrm{O}$ \\
\hline
\end{tabular}

D.E.R : dates de semis de référence ; $M x$ : date maximum ; $M$ : date moyenne ; $S D$; déviation standard.

\section{CONCLUSION}

La sécheresse agricole dans les régions semi-arides céréalières d'Algérie, montre une durée des pluies, caractérisée par un nombre de jour pluvieux restreint et par une variabilité et une 
faiblesse des cumuls de pluies supérieures à $5 \mathrm{~mm}$, de septembre à juin. Ces conditions affectent directement les échéances de dates de semis, de germinations, de fructifications et des besoins en eau au cours des phases de croissances des cultures. Ces résultats qui mettent en relief les hypothèses de départ, expliquent partiellement la production et le rendement faibles des céréales.

Une adaptation aux effets de sécheresses, s'avère cruciale pour un développement durable de la céréaliculture pluviale dans le pays.

\section{REFERENCES BIBLIOGRAPHIQUES}

[1] Smadhi D. et Zella L. Céréaliculture en sec et précipitations annuelle : le cas du Nord de l'Algérie. Rev. Sech. vol 20, $n^{\circ} 2,2009$, pp 199-203

[2] Yadh Z. Maîtrise de la croissance démographique, gestion économique de l'eau et sécurité alimentaire. Quelles perspectives d'adéquations futures en Tunisie? Sécheresse. Volume 12, Numéro 2, 2001, pp 103-10

[3] Doorembos J. et Pruitt W.O. Les besoins en eau des cultures. Bulletin d'irrigation et de drainage, $\mathrm{n}^{\circ} 24, \mathrm{FAO}, 1986,196 \mathrm{p}$.

[4] Hills R.C. et Morgan J.H.T. Rainfall statistic: an interactive approach to analysing rainfall records for agricultural purposes. Experimental agriculture 17, 1981, pp 16-16.

[5] Davey E.G., Mattei F. et Soloman S.I. En evaluation of climate and water resources for the development of agriculture in the Sudano-sahelian zone of West Africa. Geneva: Wold Meteorological Organization, 1976, 9 p.

[6] Sivakumar M.V.K. Durée et fréquence des périodes sèches en Afrique de l'Ouest. ICRISAT. Bull. Inf. $\mathrm{n}^{\circ} 13,1991,181 \mathrm{p}$.

[7] Léonard A. A. Chaînes de Markov Cachées. Essai présenté à la Faculté des études supérieures de l'Université Laval pour l'obtention du grade de maître ès sciences. Départ. Math. Stat. Fac. Sci. Gen. Uni. Laval Quebec, 2001, 45 p.

[8] Van Vyve N. Caractérisation de la variabilité spatio-temporelle de la pluie au Fakara, Niger. Mem. bio-ingénieur. Uni. Cath. Louvain. Fac. d'ingénirie biologique, agronomique et environnementale, 2006, 75 p.

[9] Sivakumar M.V.K., Maidoukia A., Stern R.D. Agroclimatologie de l'Afrique de l'Ouest : Le Niger. $2^{\text {ème }}$ édit. ICRISAT. Bull. inf. $n^{\circ} 5$, DMN. Niger, 1993, 107 p.

[10] Galloy E., Martin S., Lebreton A. Analyse des séquences de jours secs consécutifs. Application a 31 postes du réseau météorologique français. La météorologie, VIe série $\mathrm{n}^{\circ} 28$, 1982, pp 5-24. 
[11] Bergaoui Z. Contribution à l'étude statistique de la pluie dans la région de Tunis. Thèse Docteur-Ingénieur, Institut national polytechnique de Toulouse, 1983, 134 p.

[12] Benzarti Z. et Habaieb H. Etude de la persistance de la sécheresse en Tunisie par utilisation des chaînes de Markov (1909-1996). Rev. Sech. Vol 12, n4, 2, 2001, pp 15-220.

[13] Dechemi N., Ait Mouhoub D. et Souag D. Contribution à l'analyse du régime pluviométrique sur le littoral algérien. Rev. Seh. Vol 11, n¹, 510, Notes méthodologiques, 2000, pp 15-20.

[14] Meddi H. and Meddi. Etude de la persistance de la sécheresse au niveau des sept plaines algériennes par utilisation des Chaînes de Markov (1930-2003). Courrier du Savoir, n 9 , Mars 2009, pp.39-48

[15] Baldy Ch. Contribution à l'étude fréquentielle des conditions climatiques. Leur influence sur la production des principales zones céréalières d'Algérie. Document, ITGC, 1974, 72 p.

[16] Smadhi D. Régionalisation et modélisation agroclimatique en céréaliculture Pluviale. Cas du Nord de l'Algérie. Thèse Doct. Sci. Agro., ENSA, El-Harrach, Alger, 2011, 180 p.

[17] Smadhi D. et Zella L. The pluviometrical deficiencies in the pluvial cereal regions in Algeria. African Journal of Agricultural Research Vol. 7(48), 2012, pp. 6413-6420.

[18] Bouvier Ch. Etude des effets de dépendance dans une série chronologique. Application à l'étude des séquences de jours de pluies. Cahier ORSTOM, vol. XX, nº 2, 1983, pp 79-116.

[19] Muller A. Comportement asymptotique de la distribution des pluies extrêmes en France. Doct. Univ. Montpellier II. Statistique appliquées et Hydrologie, 2006, 246 p.

[20] Parent E., Bernier J. Le raisonnement bayésien. Modélisation et inférence/ Ed. Springer, Collection Statistiques et probabilités appliquées, 2007, 380 p.

[21] Heathcote R.L. Drought perception. The Environmental, economic and social significance of drought (Lovett, J.V., ed.), Sidney, Australia: Augus and Robertson, 1973, pp 17-40.

[22] El Hassani T.A. Eau, zones humides et changements climatiques. Préparation à la sécheresse et gestion des risques dans la région méditerranéenne. Document de travail sécheresse. College of agriculture and natural ressources. Inst, Agro, Vet, Hassan, Rabat, 2008, 3 p. www.doc.abhatoo.net.ma/DOC/IMG/pdf/Drought.pdf.

\section{How to cite this article:}

Smadhi D, Zella L and Bachir H. Droughts in semi-arid cereal regions of Algeria. J. Fundam. Appl. Sci., 2017, 9(2), 1063-1073. 